\title{
Zur Rolle der Zeitschrift Frühe Bildung für den wissenschaftlichen Nachwuchs
}

\section{Wahrnehmungen der Sprecher_innengruppe der PdfK- Nachwuchswissenschaftler_innen der Deutschen Gesellschaft für Erziehungswissenschaft}

\author{
Sebastian Amann¹, Bianca Bloch², Lars Burghardt ${ }^{3}$ und Magdalena Hartmann ${ }^{4}$ \\ 1 Katholische Hochschule Nordrhein-Westfalen, Paderborn \\ 2Justus-Liebig-Universität Gießen \\ ${ }^{3}$ Otto-Friedrich-Universität Bamberg \\ ${ }^{4}$ Leibniz Universität Hannover
}

\begin{abstract}
Als Sprecher_innen der Gruppe der Nachwuchswissenschaftler_innen der Kommission Pädagogik der frühen Kindheit der Deutschen Gesellschaft für Erziehungswissenschaft wurden wir anlässlich des 10-jährigen Jubiläums ${ }^{1}$ der Zeitschrift Frühe Bildung von den Herausgebenden angefragt, einen Kommentar zu der Frage zu verfassen, wie wir die Rolle der Zeitschrift für den wissenschaftlichen Nachwuchs ${ }^{2}$ wahrnehmen. Unsere nachfolgenden Ausführungen beruhen dabei auf eigenen Erfahrungen und Wahrnehmungen, die wir sowohl in unserer Position als Sprecher_innen gemacht haben, als auch als Wissenschaft-
\end{abstract}

ler_innen in Qualifizierungsphasen sowie als Vertreter_innen des Forschungsbereichs Pädagogik der frühen Kindheit.

\section{Einordnung der Zeitschrift Frühe Bildung}

Die Zeitschrift Frühe Bildung ist eine der wenigen deutschsprachigen Zeitschriften, die explizit auf das Themenfeld

\footnotetext{
Zum 10-jährigen Bestehen und dem Erfolg der Zeitschrift Frühe Bildung unsere herzlichen Glückwünsche an die Herausgeber_innen.

2 Die Bezeichnung des wissenschaftlichen Nachwuchs wird auch kritisch betrachtet und es ist nicht eindeutig, wer mit dieser Bezeichnung adressiert wird und voder sich adressiert fühlt. In diesem Artikel verwenden wir daher als Synonym auch die Bezeichnung Wissenschaftler_innen in Qualifizierungsphasen.
} 
der Pädagogik der frühen Kindheit ausgerichtet ist. In anderen Zeitschriften, in denen ebenfalls frühkindliche Themen verhandelt werden, stellen diese einen möglichen Schwerpunkt neben vielen weiteren dar. Vor diesem Hintergrund könnte geschlussfolgert werden, dass die Zeitschrift Frühe Bildung für Wissenschaftler_innen, die sich mit frühkindlichen Themen auseinandersetzen, eine zentrale und wichtige Anlaufstelle verkörpert. In der Diskussion miteinander zeigte sich jedoch, dass die Wahrnehmung der Zeitschrift auf die Fragen hin variiert, mit welchem Interesse eine Veröffentlichung in der Frühen Bildung verbunden ist und wie hoch die Chancen für die Annahme einer Einreichung gesehen werden. Im Folgenden möchten wir sowohl einige Vor- als auch Nachteile aufzeigen, die sich aus unserer miteinander geführten Debatte und aus unserer Perspektive als Wissenschaftler_innen in Qualifizierungsphasen ergeben.

Ein Vorteil stellt das aufwendige Begutachtungsverfahren dar, durch das sich die Zeitschrift Frühe Bildung auszeichnet. Veröffentlichungen, die ein Double-blindPeer-Review-Verfahren durchlaufen haben, wie es bei der Frühen Bildung eingesetzt wird, werden als qualitativ hochwertig eingeschätzt. Dies spielt vor allem (aber nicht nur) bei kumulativen Dissertations- und Habilitationsvorhaben eine entscheidende Rolle bei der Entscheidung, wo veröffentlicht wird. Des Weiteren sind die unterschiedlichen Formate des Publizierens (Schwerpunktbeiträge, Freie Beiträge, Diskussion und Innovationen) positiv hervorzuheben, da sie einen breiten Kreis an Autor_innen adressieren und sich insbesondere die Formate Diskussion und Innovationen von anderen Zeitschriftenbeiträgen absetzen. Diese bieten die Möglichkeit, neue Instrumente zu besprechen, Projekte zu erläutern, kleinere eigene Forschungsergebnisse darzustellen, aktuelle (fachliche, politische, gesellschaftliche) Diskurse bzw. Entwicklungen oder Ergebnisse bzw. Arbeiten anderer Wissenschaftler innen zu kommentieren und so neue Diskussionen zu eröffnen bzw. sich in Diskussionen sichtbar einzuarbeiten. Bei Durchsicht der bisherigen Ausgaben fällt zudem positiv auf, dass sich durch die Wahl der Heftschwerpunkte aktueller Themen der Pädagogik der frühen Kindheit angenommen wird und so neueste Forschungserkenntnisse zu einem spezifischem Thema gemeinsam in einer Ausgabe publiziert werden. Die Betreuung der jeweiligen inhaltlichen Schwerpunkte durch in diesem Bereich ausgewiesene Expert_innen ist ebenfalls positiv zu werten.

Ein Nachteil ist wiederum, dass Publikationen ausschließlich in deutscher Sprache möglich sind. Dies stellt für internationale Forschungen und einen Austausch von Forschungsergebnissen im internationalen Kontext eine Einschränkung dar. Die Option, neben deutscher Sprache auch auf englischer Sprache in der Zeitschrift veröffentlichen zu können, würde dazu beitragen, dass die eigene
Forschung international stärker wahrgenommen und rezipiert werden kann. Bei der Wahl der passenden Zeitschrift für das Publikationsvorhaben könnte diese Option dazu beitragen, dass sich für die Frühe Bildung entschieden wird.

Weiter wäre es wünschenswert, wenn die Zeitschrift einen Impact Factor ausweisen könnte. Gerade im Zuge von kumulativen Qualifikationsvorhaben legen manche Betreuende, Hochschulen oder Prüfungsordnungen hier bestimmte Kriterien fest, die eine Zeitschrift erfüllen soll, damit die dort veröffentlichten Beiträge als Teil der kumulativen Dissertation bzw. Habilitation berücksichtigt werden können. Das Fehlen des Impact Factors könnte dazu führen, dass Nachwuchswissenschaftler_innen sich ggf. für eine andere Zeitschrift entscheiden.

\section{Herausforderungen im Rahmen institutioneller Eingebundenheit}

Wissenschaftler_innen sind in ihrer Qualifizierungsphase überwiegend institutionell angebunden. Je nach (Hochschul- oder Professur-)Sozialisation werden andere Erwartungen gestellt, so dass es schwer ist, die Gesamtheit aller Wissenschaftler_innen in Qualifizierungsphasen als homogene Gruppe zu verstehen. So stellen sich unter anderem folgende Fragen, die nach Standort, spezifischer disziplinärer Ausrichtung und / oder Betreuenden eine unterschiedliche Beantwortung finden: Ist es erwünscht, hilfreich oder erstrebenswert, dass Ergebnisse der Dissertation vorab publiziert werden? Welche Unterstützungsmöglichkeiten werden vor Ort geboten? Welchen Stellenwert und welche Relevanz werden der Zeitschrift Frühe Bildung (auch durch die betreuenden Personen) zugeschrieben? Wie werden Wissenschaftler_innen in Qualifikationsphasen auf die Möglichkeit, in der Zeitschrift Frühe Bildung zu veröffentlichen, aufmerksam gemacht? Erreichen sie zum Beispiel die Call for Paper? Wissen sie überhaupt von der Chance, dort eigenständig Beiträge zur Veröffentlichung einzureichen?

Die Bedeutung der Einbindung in institutionelle Arrangements und die Anleitung am jeweiligen Hochschulstandort ist auch dann ausschlaggebend, wenn es darum geht, einen entsprechenden Beitrag einzubringen und zu positionieren. Aus unserer Sicht wird hier die Frage aufgeworfen, wie in der Wissenschaftscommunity der Pädagogik der frühen Kindheit allgemein - und in diesem Fall speziell in der Zeitschrift Frühe Bildung - die Aufgabe des Publizierens und Veröffentlichens verhandelt wird, gibt es doch keine einheitlichen Regelungen in der Deutschen Gesellschaft für Erziehungswissenschaft bzw. der Kommission Pädago- 
gik der frühen Kindheit, geschweige denn in der heterogenen deutschsprachigen Hochschullandschaft. Die Zeitschrift kann für diese Fragen als Spiegel gesehen werden, welchen Zugang und welche Anerkennung die Statusgruppe der "Nachwuchswissenschaftler_innen“ - für die im deutschsprachigen Wissenschaftsbetrieb unterrepräsentierte Disziplin der Pädagogik der frühen Kindheit - für die Publikation von Forschungsbeiträgen erhalten soll. Hier hat die Zeitschrift Frühe Bildung die Chance, einen positiven Beitrag zu leisten, indem sie noch stärker die erwünschten Publikationsmöglichkeiten für diese Statusgruppe aufzeigt und bewirbt.

Als Zwischenfazit lässt sich sagen, dass die Frühe Bildung eine der wenigen deutschsprachigen Zeitschriften mit dem expliziten Fokus auf frühkindliche Themen darstellt und damit alleine schon eine Relevanz für Wissenschaftler_innen in Qualifizierungsphasen, die in diesem Bereich forschen und arbeiten, hat - sei es als Autor_in oder als Leser_in. Die Frage nach der Rolle der Frühen Bildung für den ,wissenschaftlichen Nachwuchs“ ist allerdings nicht leicht und auch nicht eindeutig zu beantworten. Die Antwort hängt von den heterogenen und komplexen Kontexten ab, in denen Wissenschaftler innen in ihren Qualifizierungsphasen eingebunden sind. Je nachdem, wie und wo Wissenschaftler_innen institutionell angebunden sind, variieren die Anforderungen, Bedingungen und Erwartungen. Gerade für qualitative monografische Qualifikationsarbeiten ist es schwierig, vorab Teilergebnisse in einer Zeitschrift zu publizieren, da die entsprechenden Prüfungsordnungen dies teilweise gar nicht zulassen. Im Gegensatz zu kumulativen Dissertationen und Habilitationen, bei denen es vorgegeben ist, eine entsprechende Anzahl an Beiträgen vorab zu publizieren, stellt die Vorgabe, einen komplett fertigen Beitrag einzureichen, für monografische Qualifikationsarbeiten eine zusätzliche (zeitliche und organisatorische) Hürde dar, wenngleich dies keine untypische Vorgabe für die Veröffentlichung von Zeitschriftenartikeln ist. Jenseits der Qualifizierungsphasen besteht auch die Möglichkeit, über Forschungsprojekte in der Frühen Bildung zu veröffentlichen. Allerdings hängt dies dann wiederum vom jeweiligen Pro- jektteam ab, ob eine Veröffentlichung in der Frühen Bildung erwünscht ist, anerkannt und angestrebt wird. Auch wenn diese strukturellen Rahmenbedingungen Einfluss auf Veröffentlichungen in allen Zeitschriften haben, kann für die Zeitschrift zusammenfassend festgehalten werden, dass die Frühe Bildung in unserer Wahrnehmung vorzugsweise Wissenschaftler_innen mit kumulativen Qualifikationsvorhaben (und dort auch nur unter bestimmten Voraussetzungen), vor allem in der Post-Doc-Phase und im Kontext von (Drittmittel-) Forschungsprojekten eine erreichbare Plattform bietet, um wissenschaftliche Ergebnisse zu publizieren.

\section{Wünsche und Anregungen}

Wir würden uns für Wissenschaftler_innen mit quantitativen Forschungsdesigns wünschen, dass die Zeitschrift Frühe Bildung einen offiziellen Impact Factor ausweist. Ebenfalls wären Publikationsmöglichkeiten in englischer Sprache wünschenswert, um den Kreis der Leser_innenschaft zu erweitern und auch, um an Vorgaben sowie Anforderungen bei Veröffentlichungen von kumulativen Qualifiaktionsvorhaben anschließen zu können. Darüber hinaus wäre eine gezieltere Ansprache und Ermutigung von Wissenschaftler_innen in Qualifizierungsphasen, einen Beitrag einzureichen, wünschenswert, um den Stellenwert und die Bekanntheit der Zeitschrift in dieser Statusgruppe zu erhöhen.

\footnotetext{
Verfasser_innen: Lars Burghardt, Otto-Friedrich-Universität Bamberg, lars.burghardt@uni-bamberg.de; Sebastian Amann, Katholische Hochschule Nordrhein-Westfalen, s.amann@kathonrw.de; Bianca Bloch, Justus-Liebig-Universität Gießen, bianca.c.bloch@erziehung.uni-giessen.de; Magdalena Hartmann, Leibniz Universität Hannover, magdalena.sophie.hartmann@ifs. uni-hannover.de
}

https://doi.org/10.1026/2191-9186/a000548 